\title{
New dimensionless indices of structural habitat complexity: predicted and actual effects on a predator's foraging success
}

\author{
Aaron Bartholomew*, Robert J. Diaz, Giancarlo Cicchetti
}

Virginia Institute of Marine Science, Gloucester Point, Virginia 23062, USA

\begin{abstract}
Ecologists have long known that complex habitats often provide prey with refuges from predation. This is true for a wide variety of habitat types in terrestrial, freshwater and marine systems. Despite the recognized importance of structural habitat complexity, ecologists have defined and measured complexity in many different ways. We propose 2 new indices of structural habitat complexity that are dimensionless, that can be applied across various habitat types and scales, and that directly measure how structural complexity interferes with a predator's foraging ability. These indices are: the total area of cover within a habitat divided by the total area of the habitat $\left(C_{t} / A_{t}\right)$ and the average inter-structural space size divided by the size of the predator $(\mathrm{Sp} / \mathrm{Pr}) . \mathrm{C}_{t} / \mathrm{A}_{t}$ measures the amount of cover available within a habitat that interferes with a predator's ability to see, or otherwise sense, prey within the habitat. Sp/Pr measures the extent to which the structure interferes with a predator's ability to move through the habitat in search of, or while pursuing, prey. We predicted that prey survivorship should increase hyperbolically with increasing $C_{t} / A_{t}$ and that survivorship should decrease sigmoidally with increasing Sp/Pr. We also predict that both $C_{t} / A_{t}$ and $S p / P r$ can influence survivorship independently, and that they form a survivorship plane. We tested our model in 3 laboratory experiments with the fish Fundulus heteroclitus as predator and amphipods as prey, and in 1 field experiment. The results of our laboratory experiments support our model for $\mathrm{Sp} / \mathrm{Pr}$, but are only suggestive for $C_{t} / A_{t}$. The results of the field experiment are consistent with our laboratory results, and our model.
\end{abstract}

KEY WORDS: Habitat complexity · Habitat structure $\cdot$ Dimensionless indices $\cdot$ Predation $\cdot$ Fundulus heteroclitus · Amphipods

Resale or republication not permitted without written consent of the publisher

\section{INTRODUCTION}

Complex habitats often have both greater faunal abundances and diversities than nearby, simpler habitats. Mechanisms that cause these patterns include, but are not limited to decreased physical stress (Dean \& Connell 1987), increased food availability (Hicks 1985, Gorham \& Alevizon 1989), increased resource or niche availability (O'Connor 1991), increased amounts of surface area for living (Heck \& Wetstone 1977), decreased competition (Diehl 1988), and increased refuges from predation (Orth et al. 1984) in complex

*E-mail: bart@vims.edu habitats. In the literature, ecologists have focused most attention on the effect that complex habitats have on predator/prey relationships. Ecologists have demonstrated that complex habitats provide refuges from predation in a wide variety of terrestrial, freshwater and marine habitat types, and for various fauna, including mammals (Dickman 1992), insects (Pierce 1988), birds (Schneider 1984), fish (Werner \& Hall 1988), crustaceans (Jordan et al. 1996), and mollusks (Aronson 1986).

Despite the recognized importance of habitat complexity, ecologists remain somewhat confused about how to best define and measure structural complexity (Lipcius et al. 1998). For example, marine ecologists have often measured specific aspects of a particular 
habitat type as complexity: Spartina alterniflora culm density (van Dolah 1978), number of seagrass blades $\mathrm{m}^{-2}$ (James \& Heck 1994) and amount of shell material in polychaete worm tubes (Bell 1985). These measurements may be quite useful within their specific habitat types, but they make comparison of results between studies difficult. Researchers have also used more universally applicable complexity indices, including biomass, surface area (Heck \& Crowder 1991), and surface area to volume ratios (Coull \& Wells 1983). Prey survivorship tends to increase as these habitat parameters increase, but not always (Heck \& Crowder 1991). Furthermore, studies using these complexity indices do not always explain why the habitat parameter employed should directly affect prey survivorship.

These complexity indices also fail to address the measurement scale involved, which McCoy \& Bell (1991) state is an important component of habitat structure. Complex habitats function as predation refuges across a variety of faunal scales, from macrofauna (Heck \& Wetstone 1977) to meiofauna (Coull \& Wells 1983), and complexity indices should allow comparisons of results between scales.

McCoy \& Bell (1991) state that 'habitat structure' encompasses the absolute abundance of habitat structural components (complexity), the relative abundances of different habitat structural components (heterogeneity), and the scale used to measure these. In this paper we devise 2 new indices of habitat complexity, as defined by McCoy \& Bell (1991), that address the effect of complexity on predator foraging success and prey survival. These indices incorporate the measurement scale in a way that makes them dimensionless, and they can be applied to any habitat scale and type.

Complex habitats provide refuges for prey by limiting a predator's ability to move throughout a habitat in search of prey, by interfering with a predator's ability to detect prey within the habitat, and by interfering with a predator's ability to catch prey, once detected (Ryer 1988, James \& Heck 1994). We propose 2 new structural habitat indices that directly measure these interference mechanisms: (1) the total amount of crosssectional area (total cover) within a given habitat area for prey to hide behind, and (2) the sizes of the interstructural spaces or gaps that the predator must maneuver through relative to the size of the predator itself.

To account for predators' reduced maneuverability in complex habitats, we propose measuring the average inter-structural space size within the habitat of interest (Sp). One can then divide Sp by the predator's size (Pr), to scale it to the predator of interest. Pr is the largest linear distance perpendicular to the predator's normal direction of motion, and perpendicular to the dominant orientation, if any, of the structure within the habitat. $\mathrm{Sp} / \mathrm{Pr}$ is dimensionless, and it measures the extent to which the spaces within the habitat restrict a given-sized predator's movement.

We propose that at $\mathrm{Sp} / \mathrm{Pr}$ values below 1, the predator cannot move through the habitat on average, and prey survivorship should be uniformly high. As the average $\mathrm{Sp} / \mathrm{Pr}$ increases beyond 1, the number of spaces that the predator can fit through accumulates, the predator's maneuverability within these spaces also increases, and prey survivorship should decrease rapidly. Finally, Sp/Pr should reach a point where the predator's maneuverability is no longer hindered, and prey survivorship should be uniformly low. Overall, this forms a decreasing sigmoid, or 'threshold', curve with increasing Sp/Pr.

Complexity indices should also account for the predator's reduced ability to see or otherwise sense prey in complex habitats. Stoner (1980) found that surface area predicted prey survivorship better than biomass (volume) of substrate as proposed by Heck \& Wetstone (1977). However, given equal biomasses of 3 seagrass species, Stoner (1982) later found that the species with the highest surface area provided the worst refuge for an amphipod prey, as predators could easily detect the prey through gaps in the small branches. We propose that total structural cross-sectional area, or total 'cover', may better measure how structure interferes with a predator's ability to detect prey.

The cover provided by an individual structure is the largest cross-sectional area of that structure, similar to viewing the structure as a shadow, and it measures the amount of area within the habitat that the structure obstructs from view. One can then divide the total cover $\left(C_{t}\right)$ by the total area of the habitat of interest $\left(A_{t}\right)$ to create a dimensionless measure of the amount of cover within a given area.

We propose that increasing $C_{t} / A_{t}$ will result in hyperbolically increasing prey survivorship. We propose a hyperbolic curve because, as the amount of structure within a habitat increases, the functional redundancy of each new structure, in terms of sensory obstruction, also increases. For example, imagine a habitat containing a single structure. Adding another structure obstructs more of the habitat from sensory detection. However, the new structure also obstructs part of the habitat already blocked off by the original structure, and vice versa, making the 2 structures functionally redundant for some parts of the habitat. As more structures are added (increasing $C_{t} / A_{t}$ ), we get continually diminished returns in the amount of additional habitat that is obstructed from sensory detection, and thus continually diminished returns in prey survivorship. Overall, this implies a hyperbolic relationship of $C_{t} / A_{t}$ and prey survivorship.

$\mathrm{C}_{\mathrm{t}}$ and Sp size can vary independently of each other, and each can contribute to increased prey survivor- 
ship. We propose that prey survivorship varies as a function of both $\mathrm{C}_{t} / \mathrm{A}_{\mathrm{t}}$ and $\mathrm{Sp} / \mathrm{Pr}$, forming a survivorship plane similar to that in Fig. 1. In this conceptual model, adding cover to a habitat with a large $\mathrm{Sp} / \mathrm{Pr}$ value increases prey survivorship hyperbolically, but adding cover to a habitat with small $\mathrm{Sp} / \mathrm{Pr}$ values has little impact, as prey survivorship is already high. Similarly, decreasing $\mathrm{Sp} / \mathrm{Pr}$ values in a habitat with little cover increases prey survivorship in a sigmoid or threshold manner, whereas decreasing $\mathrm{Sp} / \mathrm{Pr}$ values in a habitat with adequate cover would have less impact. To test this model we performed 3 laboratory experiments, and 1 field experiment.

\section{MATERIALS AND METHODS}

First laboratory experiment. We performed this experiment to determine the general relationship between prey survivorship and increasing $\mathrm{Sp} / \mathrm{Pr}$ and $\mathrm{C}_{t} / \mathrm{A}_{\mathrm{t}}$, and to test our results against our model. Larger individuals ( $>8 \mathrm{~cm}$ length) of the fish Fundulus heteroclitus were the predators for all 3 laboratory experiments. We chose fish $>8 \mathrm{~cm}$ in length to ensure that they were not gape limited for the amphipods we used (authors' pers. obs.). F. heteroclitus are ubiquitous inhabitants of Spartina alterniflora salt marshes along the east coast of the United States. They are omnivorous, and have been previously used in predation experiments with amphipods (Van Dolah 1978). We collected fish for all 3 laboratory experiments with

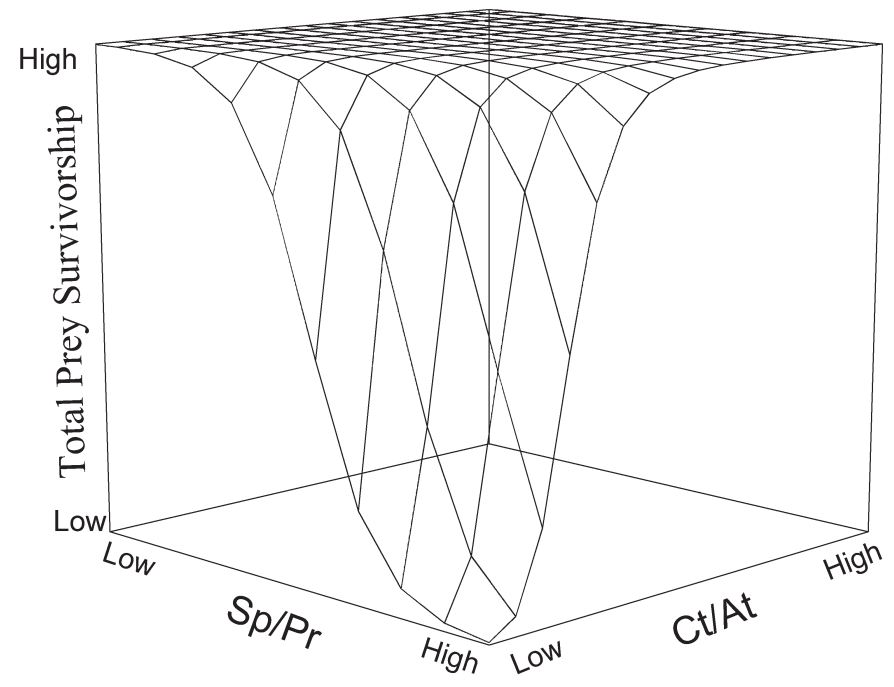

Fig. 1. Survivorship model. Hypothetical plane with total prey survivorship as a function of total cover area/total habitat area $\left(\mathrm{C}_{\mathrm{t}} / \mathrm{A}_{\mathrm{t}}\right)$ and average space size/prey size (Sp/Pr). Total prey survivorship increases hyperbolically with increasing $C_{t} / A_{t}$, and increases sigmoidally with decreasing $\mathrm{Sp} / \mathrm{Pr}$ minnow traps from salt marshes adjacent to the York River, Virginia. For the first experiment, the fish were collected in late October 1997 and kept for $3 \mathrm{wk}$ prior to experimentation in a large, outdoor, flow-through tank with water from the York River. In all 3 laboratory experiments the fish were fed commercial flake food.

We used larger sized individuals of the amphipod Leptocheirus plumulosus as our prey in the first and second laboratory experiments. L. plumulosus is normally a tube dweller on muddy bottoms (Bousfield 1973). When it does not have mud to burrow in (as in these experiments), it moves about, mostly crawling on the bottom. When disturbed, it exhibits a quick, swimming escape response (authors' pers. obs.), and for the purposes of these experiments it is considered a mobile prey item. We obtained L. plumulosus from cultures maintained at the Virginia Institute of Marine Science.

We conducted all 3 laboratory experiments in a Virginia Institute of Marine Science wet lab. This lab has flow-through estuarine water from the York River and climate control. We placed the experimental tanks in a row on the floor, and filled each with York River water approximately $20 \mathrm{~h}$ before experimentation. We randomly assigned each complexity treatment to a container for each run, during all 3 experiments. Twentyfour hours before experimentation, we placed fish in a separate, empty container for starvation. Two hours before experimentation 16 amphipods were randomly added to each container; we also placed 2 randomly selected fish in the water suspended by mesh bags from openings in the tops. This allowed both the fish and the amphipods to acclimate, without the fish eating the amphipods. Each tank had 2 fish because single fish did not consistently feed in pilot studies.

The experiments began when the fish were released to feed on the amphipods. We released fish in a random order, and recaptured them in the same order. The elapsed time to release the fish was about $2 \mathrm{~min}$, and to recapture about $5 \mathrm{~min}$. We were careful to minimize disturbance to the fish throughout the experiment, as disturbed fish did not feed for 10 to $15 \mathrm{~min}$ after disturbance (authors' pers. obs.). We stopped the experiment 75 min after release by quickly disturbing each tank by tapping on the side. We then captured each fish with a dip net, placed them in bags for later measurement, and counted surviving amphipods. We measured the gill- to-gill width of the fish, as this dimension would affect the fish's maneuverability within the tanks. The average linear space between the vertically oriented dowels was limiting, so we measured the fish's largest horizontal linear distance perpendicular to the dowels, and to the fish's normal direction of motion (gill-to-gill width for Fundulus heteroclitus).

Amount of prey and exposure times were not arbitrarily chosen in these experiments. We used 16 am- 
phipods because the fish could eat this amount without apparent satiation. We chose 75 min exposure times because in preliminary tests it took about this long for 2 starved Fundulus heteroclitus to eat 16 amphipods in our zero structure treatment. By using 16 amphipods exposed for $75 \mathrm{~min}$, we are fairly certain that any prey survivorship we observed was due to the structural complexity alone. For the first experiment, we performed 6 runs over $9 d_{i}$ for the second experiment, we performed 9 runs over $14 d_{i}$ and for the third experiment we performed 6 runs over $11 \mathrm{~d}$.

In the first laboratory experiment we had tanks with 6 different complexity levels formed by evenly spaced, $0.30 \mathrm{~cm}$ diameter wooden dowels, and 1 tank with no dowels. The tanks for all the laboratory experiments were $36 \times 94 \times 22 \mathrm{~cm}$ and plastic, filled to a depth of $14.8 \mathrm{~cm}$. The tanks had rounded edges, to minimize potential corner effects, and we covered the exterior sides and bottoms with white paper, which allowed light in while preventing outside motion from disturbing the fish. The wooden dowels creating the structure protruded downward, almost perpendicularly, through the tank tops until they touched the bottoms. In the middle of each tank top was an $8 \times 8 \mathrm{~cm}$ square opening, through which we could place amphipods and fish into the tanks, and which allowed light in. During the experiment these 'opening' portions of the tanks remained without dowels, but they represented only $2 \%$ of the total water volume. The habitat structural parameters for this experiment are shown in Table 1.

The null hypothesis for the first laboratory experiment was: there is no relationship between either $C_{t} / A_{t}$ or $\mathrm{Sp} / \mathrm{Pr}$ and prey survivorship. In this first experiment, biomass (volume), number (density) and surface area of dowels all covaried exactly with the total cover of the dowels, so we could not determine which of these habitat parameters best predicted prey survivorship. We tested the first null hypothesis using logistic regression with SAS, similar to Gotceitas \& Colgan (1989). This statistical procedure treats each amphipod as an independent bernoulli trial with 2 possible categorical outcomes: 'survived' or 'eaten' (Agresti 1990), and the survivorship results from all experimental runs were pooled. In this approach each individual amphipod is a replicate (Agresti 1990).

We analyzed the curve shapes of $\mathrm{C}_{t} / \mathrm{A}_{\mathrm{t}}$ and $\mathrm{Sp} / \mathrm{Pr}$ versus prey survivorship by estimating the first derivatives of the curves, an approach suggested by Lipcius et al. (1998). We calculated the first derivatives for all the data points as: $\Delta$ survivorship divided by $\Delta \mathrm{Sp} / \mathrm{Pr}$ or $\Delta C_{t} / A_{t}$ and then plotted these values versus $S p / \operatorname{Pr}$ or $\mathrm{Ct} / \mathrm{At}$, respectively. The midpoints of each of the 2 points used were the $x$ coordinates. We proposed a negatively sloped sigmoid relationship between $\mathrm{Sp} / \mathrm{Pr}$ and survivorship, and the first derivative shape for this relationship would resemble a ' $\mathrm{V}$ ' or ' $\mathrm{U}$ ' with all values being negative (Lipcius et al. 1998). We proposed a positive hyperbolic relationship between $C_{t} / A_{t}$ and survivorship, and the first derivative shape for this relationship would be higher, positive values at low $\mathrm{C}_{t} / \mathrm{A}_{t}$ values, with values dropping rapidly before leveling off at lower, positive values at high $\mathrm{C}_{\mathrm{t}} / \mathrm{A}_{\mathrm{t}}$ (Lipcius et al. 1998). The first derivative of a simple, linear relationship of survivorship versus $S p / \operatorname{Pr}$ or $C_{t} / A_{t}$ would be a flat line (Lipcius et al. 1998).

Second laboratory experiment. We performed this experiment to determine whether $C_{t} / A_{t}$ and $S p / P r$ influence prey survivorship independently, and in particular whether increased $C_{t} / A_{t}$ within equal $S p / P r$ levels, leads to increased prey survivorship. This experiment also allows us to determine whether $C_{t} / A_{t}$ biomass (volume) of dowels or number (density) of dowels best predicted prey survivorship, as these parameters did not covary exactly as in Expt 1 . We could not make this distinction for total dowel surface area, as this parameter still covaried exactly with $C_{t} / A_{t}$.

For this experiment the predator and prey were the same as the first experiment. We collected the fish in early January 1998, and kept them for between 1 and $9 \mathrm{~d}$ prior to experimentation in a large, indoor, flowthrough tank. We created 4 different complexity treatments, formed by evenly spaced 0.30 or $0.95 \mathrm{~cm}$ diameter wooden dowels. Two tanks had almost equal, wide, average space sizes. We constructed one of these with small dowels, and the other with large dowels, so they each contained different amounts of total cover. The other 2 tanks had almost equal, narrow, average space sizes, again constructed with different sized dowels, producing different amounts of cover. The habitat structural parameters for this experiment are shown in Table 3.

The second null hypothesis was: survivorship is independent of both space size and dowel size. We tested this null hypotheses using log-linear modeling with SAS, and survivorship results from each run were again pooled for this analysis. If survivorship was independent of dowel size, but not of space size, we would conclude that differences in $C_{t} / A_{t}$ between the treatments were not important to survivorship, but that differences in $\mathrm{Sp} / \mathrm{Pr}$ were important. We graphed the first derivative of $C_{t} / A_{t}$ and survivorship versus $C_{t} / A_{t}$ as described in the first laboratory experiment.

Third laboratory experiment. We performed this experiment to again determine whether $C_{t} / A_{t}$ and $\mathrm{Sp} / \mathrm{Pr}$ influence prey survivorship independently, and in particular whether decreased $\mathrm{Sp} / \mathrm{Pr}$, within equal $\mathrm{C}_{\mathrm{t}} / \mathrm{A}_{\mathrm{t}}$ treatments, leads to increased prey survivorship. We had 6 treatment combinations with 3 cover levels (low, medium and high) and 2 sizes of predator (larger and smaller). In this way there were different $\mathrm{Sp} / \mathrm{Pr}$ 
values within a single cover level, due to the gill-to-gill width differences of the fish.

For the third experiment we collected fish in early June 1998, and kept them between 1 and 11 d before experimentation in a large, indoor, flow-through tank. The prey for this experiment were larger individuals of the amphipod Gammarus mucronatus, common epifaunal amphipods, which we collected from the York River, Virginia. We used different amphipod species in the 3 experiments because of species availability.

We constructed pairs of low, medium, and high cover treatments, such that each member of a pair had the same total cover and average inter-structural space size. The structure was formed by evenly spaced $0.30 \mathrm{~cm}$ diameter dowels. The habitat structural parameters for this experiment are shown in Table 5. Each member of a pair received a different sized fish, either less than or equal to $1.3 \mathrm{~cm}$ gill-to-gill width or greater than or equal to $1.5 \mathrm{~cm}$ gill-to-gill width, so that each container had different $\mathrm{Sp} / \mathrm{Pr}$ values.

The third null hypothesis was: survivorship is independent of both fish size and cover level. We tested this hypothesis using log-linear modeling in SAS, and survivorship results from each run were again pooled for this analysis. If survivorship was not independent of cover level, but was independent of fish size, then we would conclude that $\mathrm{Sp} / \mathrm{Pr}$ was not important to survivorship, but that $C_{t} / A_{t}$ was important. We graphed the first derivative for $\mathrm{Sp} / \mathrm{Pr}$ and survivorship versus $\mathrm{Sp} / \mathrm{Pr}$, as described in the first laboratory experiment.

Field experimental procedure. We performed this experiment to determine if differences in space sizes observed in nature possibly affected the size distribution of fish. If large Fundulus heteroclitus were less maneuverable in small spaces in our lab experiments, we might expect that larger $F$. heteroclitus would be less likely to be found in areas with smaller inter-structural spaces, and thus smaller $\mathrm{Sp} / \mathrm{Pr}$ values.

The field site was part of the Goodwin Islands, located at the mouth of the York River $\left(37^{\circ} 12^{\prime} 46^{\prime \prime} \mathrm{N}\right.$, $\left.76^{\circ} 23^{\prime} 46^{\prime \prime} \mathrm{W}\right)$. In late July 1998, we measured interstructural space distances at 70 randomly chosen points in both short $(<45 \mathrm{~cm}$ height), and tall $(>45 \mathrm{~cm}$ height) form Spartina alterniflora. Short form $S$. alterniflora is generally found higher on the marsh surface than tall form $S$. alterniflora, and both forms are readily distinguishable on this marsh. The short form $S$. alterniflora appears to be more closely spaced than the tall form $S$. alterniflora. At each point we haphazardly chose a single $S$. alterniflora plant and measured, at $12 \mathrm{~cm}$ above the marsh surface, the distance from the plant to the nearest structure within a randomly chosen $90^{\circ}$ arc of the plant. We did this to determine if there was a significant difference in space size between short versus tall form $S$. alterniflora. We tested the null hypothesis that there was no difference in inter-structural space size between short and tall form $S$. alterniflora with a 2-tailed $t$-test. Space sizes were log transformed to achieve normality.

If we found a difference in space sizes between the Spartina forms, we would test the null hypothesis: there is no difference between fish sizes caught within these different forms of $S$. alterniflora, controlling for seasonal and depth effects. Fish size data within the 2 $S$. alterniflora forms came from daylight $1.48 \mathrm{~m}$ diameter drop ring samples previously collected at this site (Cicchetti 1998). We had 29 independent samples from short form $S$. alterniflora, and 36 independent samples from tall form $S$. alterniflora. Sampling occurred from June to November 1995, and May to September 1996. The recovery efficiency for Fundulus heteroclitus from 2.8 to $10.2 \mathrm{~cm}$ length with this gear in S. alterniflora is $84 \%$ (Cicchetti 1998).

We tested the null hypothesis of no difference in fish sizes between Spartina alterniflora forms using ANCOVA, with 'time of year' and water depth as covariates. For 'time of year' both 1995 and 1996 data were combined after a separate ANCOVA demonstrated no size difference between years. 'Time of year' was defined as follows: the sampling date earliest in the year (in this case May 6, 1996) was assigned the value Day 1, and subsequent dates were numbered accordingly (Day 199 was the latest day in the year, November 21, 1995). We used time of year as a covariate because Chesapeake Bay Fundulus heteroclitus recruits first appear in the spring and grow throughout the year, so fish size would tend to be smaller earlier in the year, just as $S$. alterniflora height would tend to be shorter. The water depth $(\mathrm{cm})$ was measured in the drop ring at each drop. We selected water depth as a covariate because there is evidence that small $F$. heteroclitus may go into shallower water than larger $F$. heteroclitus (Ruiz et al. 1993), and short form S. alterniflora tends to be higher on the marsh surface, and thus in shallower water when the marsh is flooded.

Fish sizes were recorded as wet weights (g) by Cicchetti (1998). We converted grams of wet weight to gill-to-gill width $(\mathrm{cm})$ using the equation:

gill-to-gill width $=\frac{0.7397 \text { (wet weight) }}{0.1903+(\text { wet weight })}+0.0726$ (wet weight)

(adjusted $\mathrm{r}^{2}=0.98$, the regression is highly significant $\mathrm{p}<0.0001$ ). To obtain this equation we measured gillto-gill width of $80 \mathrm{~F}$. heteroclitus from the York River, all between 0.08 and $11.10 \mathrm{~g}$ wet weight. The fish sizes used in the ANCOVA were converted average F. heteroclitus gill-to-gill width within each independent drop ring sample. 


\section{RESULTS}

\section{First laboratory experiment}

For Expt 1 the log odds of amphipod survival = $-0.4631-0.5116(\mathrm{Sp} / \mathrm{Pr})+2.4387\left(\mathrm{C}_{\mathrm{t}} / \mathrm{A}_{\mathrm{t}}\right)$. The chi-square value was $10.8(\mathrm{p}=0.001)$ for the $\mathrm{Sp} / \mathrm{Pr}$ coefficient, and $17.4(p=0.0001)$ for the $C_{t} / A_{t}$ coefficient. This indicated significant relationships of survivorship, the dependent variable, with both $S p / P r$ and $C_{t} / A_{t}$, the independent variables. Total surface area, number (density) of dowels and biomass (volume) of dowels also would have generated significant results similar to $C_{t} / A_{t}$ since these parameters all covaried exactly (Table 1). The Pearson chi-square for this equation, a goodnessof-fit statistic, was 0.36 with 4 degrees of freedom, indicating an adequate fit $(p=0.99)$ of the equation to the data (Stokes et al. 1995). The relationship between $\mathrm{Sp} / \mathrm{Pr}$ and survivorship was obviously non-linear (Fig. 2a), but did not conform to a negative sigmoid relationship (Fig. 2c). The relationship between $C_{t} / A_{t}$ and survivorship was certainly positive (Fig. 2b). The exact shape of the curve is problematic, however (Fig. 2d). The small peak seen in this plot indicates that the relationship may be slightly sigmoid (Lipcius et al. 1998); however the peak's small magnitude may indicate a simple linear relationship. Surface area/volume remained constant throughout this experiment and was a poor predictor of prey survivorship.

\section{Second laboratory experiment}

The most parsimonious log-linear model for the second experimental results indicated that survivorship was significantly non-independent of both space size and dowel size (Table 2). The results indicate that survivorship from treatments with large dowels was higher than those with small dowels, despite nearly identical $\mathrm{Sp} / \mathrm{Pr}$ values (Fig. 3a). The relationship between $C_{t} / A_{t}$ and survivorship appears to be simply linear (Fig. 3b,c). Neither volume (biomass) nor number (density) of dowels demonstrated a clear pattern with prey survivorship in the second experiment (Table 3). Total surface area had the same pattern as $\mathrm{C}_{\mathrm{t}} / \mathrm{A}_{\mathrm{t}}$, as they again covaried exactly (Table 3 ).

\section{Third laboratory experiment}

The most parsimonious log-linear model for the Expt 3 results indicated that survivorship was significantly non-independent of both fish size and cover level (Table 4). Survivorship versus $\mathrm{Sp} / \mathrm{Pr}$ followed a pattern similar to the first experiment (Fig. 4a). The results
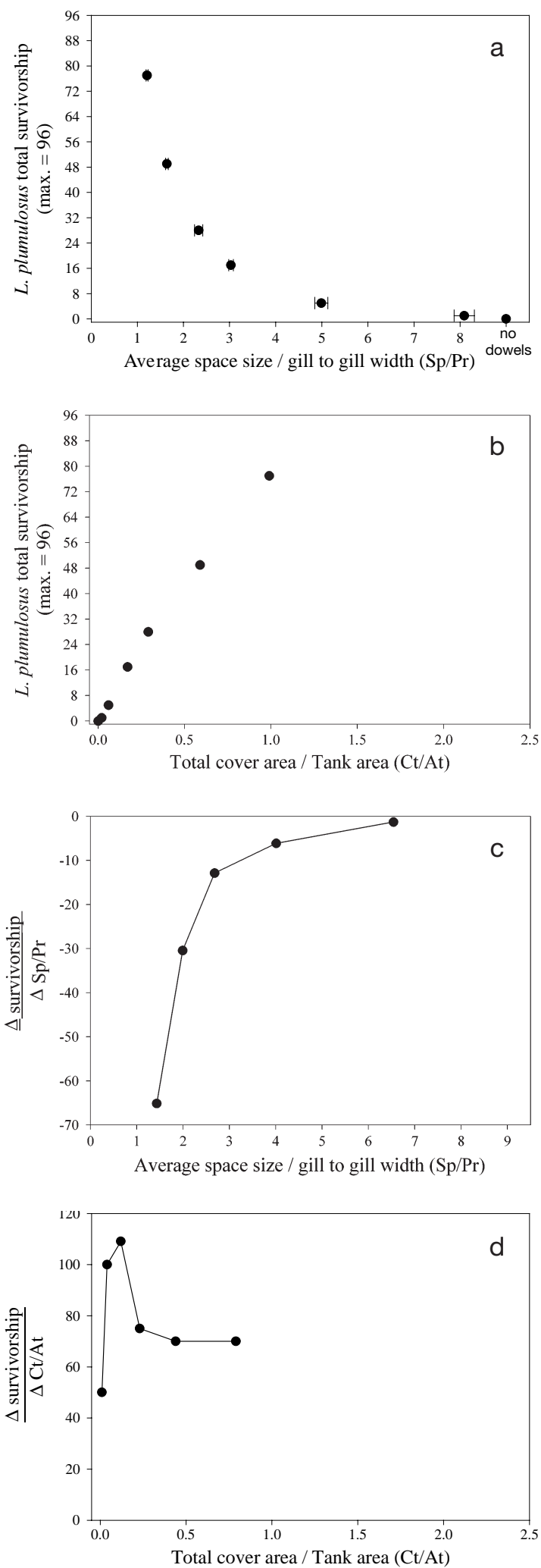

Fig. 2. Results of Expt 1. (a) Total prey (Leptocheirus plumulosus) survivorship versus $\mathrm{Sp} / \mathrm{Pr}$; error bars = $1 \mathrm{SE}$. (b) Total prey survivorship versus $C_{t} / A_{t}$. (c) First derivative of (a) versus Sp/Pr. (d) First derivative of (b) versus $C_{t} / A_{t}$ 

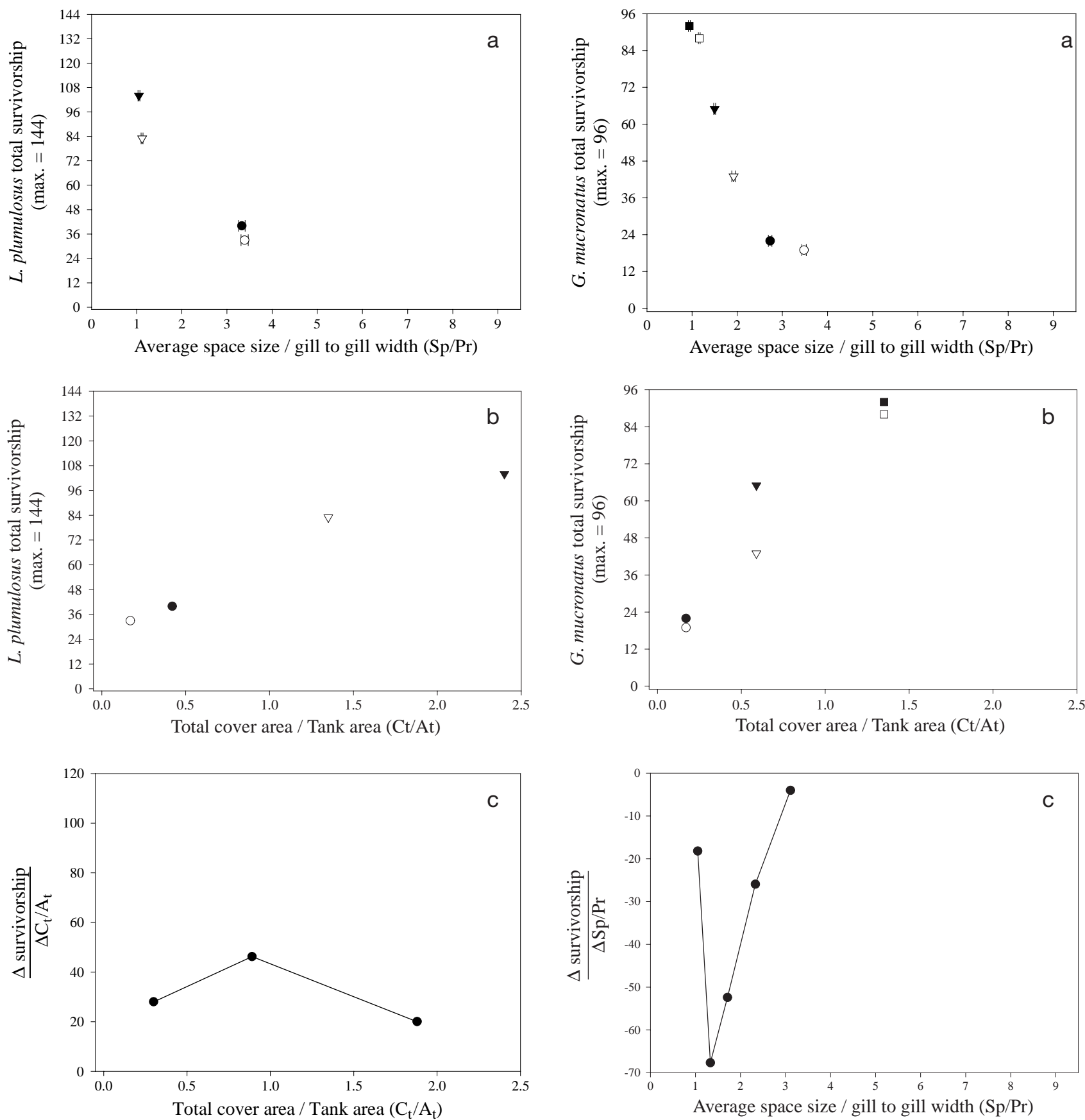

Fig. 3. Results of Expt 2. (a) Total prey (Leptocheirus plumulosus) survivorship versus $\mathrm{Sp} / \mathrm{Pr}$; error bars = $1 \mathrm{SE}$. (b) Total prey survivorship versus $C_{t} / A_{t}$. Triangles are narrow space treatments, circles are wide-space treatments, filled shapes are large dowel treatments, and open shapes are small dowel treatments for both (a) and (b). (c) First derivative of (b) versus $C_{t} / A_{t}$

indicated a sigmoid relationship (Fig. 4c), with the start of a high survivorship plateau at very low levels of $\mathrm{Sp} / \mathrm{Pr}$, and with a low survivorship plateau at higher $\mathrm{Sp} / \mathrm{Pr}$ levels. The pattern of survivorship with increas-

Fig. 4. Results of Expt 3. (a) Total prey (Gammarus mucronatus) survivorship versus $\mathrm{Sp} / \mathrm{Pr}$; error bars = $1 \mathrm{SE}$. (b) Total prey survivorship versus $C_{t} / A_{t}$. Squares are high cover treatments, triangles are medium cover treatments and circles are low cover treatments, filled shapes are large fish treatments, and open shapes are small fish treatments for both (a) and (b).

(c) First derivative of (a) versus $\mathrm{Sp} / \mathrm{Pr}$

ing $C_{t} / A_{t}$ (Fig. 4b) indicates that prey survivorship is higher for larger fish treatments, particularly from the medium cover treatments. The medium cover treatments spanned the threshold area of Fig. 4a. Table 5 
Table 1. Expt 1 results and structural parameters for the containers

\begin{tabular}{|c|c|c|c|c|c|c|c|}
\hline & & & \multirow{2}{*}{\multicolumn{3}{|c|}{$\begin{array}{c}\text { Complexity treatment } \\
3\end{array}$}} & \multirow[b]{2}{*}{5} & \multirow[b]{2}{*}{6} \\
\hline & 0 & 1 & & & 4 & & \\
\hline $\begin{array}{l}\text { Total amphipod survivors } \\
\quad(\text { max. survival }=96)\end{array}$ & 0 & 1 & 5 & 17 & 28 & 49 & 77 \\
\hline $\begin{array}{l}\text { Avg. amphipod surviviorship } \\
\text { per run (max. = 16) (SE) }\end{array}$ & $0(0)$ & $0.17(0.17)$ & $0.83(0.65)$ & $2.83(1.54)$ & $4.67(1.50)$ & $8.17(2.56)$ & $12.83(1.14)$ \\
\hline Avg. fish size, cm (SE) & $1.41(0.04)$ & $1.49(0.04)$ & $1.43(0.04)$ & $1.43(0.02)$ & $1.47(0.05)$ & $1.43(0.03)$ & $1.45(0.03)$ \\
\hline Between-dowel space size $(\mathrm{cm})$ & - & 11.97 & 7.06 & 4.30 & 3.38 & 2.33 & 1.74 \\
\hline $\mathrm{Sp} / \mathrm{Pr}(\mathrm{SE})$ & - & $8.09(0.22)$ & $4.99(0.14)$ & $3.03(0.05)$ & $2.33(0.09)$ & $1.64(0.03)$ & $1.21(0.02)$ \\
\hline Total cover area $\left(\mathrm{cm}^{2}\right)$ & 0 & 62 & 213 & 590 & 999 & 2020 & 3397 \\
\hline $\mathrm{C}_{\mathrm{t}} / \mathrm{A}_{\mathrm{t}}$ & 0 & 0.02 & 0.06 & 0.17 & 0.29 & 0.59 & 0.99 \\
\hline $\begin{array}{l}\text { Total volume of dowels }\left(\mathrm{cm}^{3}\right) \\
\text { (proportional to biomass) }\end{array}$ & 0 & 15 & 50 & 139 & 235 & 476 & 800 \\
\hline $\begin{array}{l}\text { Total number of dowels } \\
\text { (proportional to density) }\end{array}$ & 0 & 14 & 48 & 133 & 225 & 455 & 765 \\
\hline Total surface area of dowels $\left(\mathrm{cm}^{2}\right)$ & 0 & 195 & 670 & 1855 & 3138 & 6347 & 10671 \\
\hline
\end{tabular}

indicates the survivorship pattern with various habitat parameters for the third experiment. Overall, the survivorship patterns were fairly consistent between experiments, particularly between the first and third experiments, despite different experimental dates and the use of 2 different prey species.

\section{Field experiment}

We found that there was a small but significant difference in space size between short and tall form Spartina alterniflora $(t=2.16$, $\mathrm{df}=137, \mathrm{p}=0.032)$. The average space sizes were 4.0 and $5.0 \mathrm{~cm}$ for short and tall form $S$. alterniflora, respectively. Both means were highly influenced by rarer, large spaces, and it might be more instructive to examine the median space sizes in each

Table 2. Maximum likelihood log-linear model for Expt 2. Note: The interactions between dowel size and prey survival, and space size and prey survival test the null hypotheses that these factors are independent. A significant result indicates non-independence

\begin{tabular}{|lccc|}
\hline Source & df & Chi-square & $\mathrm{p}$ \\
\hline Dowel size & 1 & 0.05 & 0.8189 \\
Space size & 1 & 1.02 & 0.3125 \\
Survival & 1 & 6.46 & 0.0110 \\
Dowel size $\times$ Survival & 1 & 5.48 & 0.0193 \\
Space size $\times$ Survival & 1 & 85.63 & $<0.00005$ \\
Likelihood ratio & 2 & 2.16 & 0.3402 \\
\hline
\end{tabular}

form, which were 3.3 and $4.2 \mathrm{~cm}$ for short and long form $S$. alterniflora, respectively. The ANCOVA results on the field data from Cicchetti (1998) indicate a significant positive relationship of fish size with the covariates date (Fig. 5a) and water depth (Fig. 5b), but no relationship with the factor $S$. alterniflora form (Table 6).

\section{DISCUSSION}

Based on first principles, we predicted that prey survivorship would decrease sigmoidally with increasing average space size/predator width $(\mathrm{Sp} / \mathrm{Pr})$, and increase hyperbolically with increasing cover area/tank area $\left(C_{t} / A_{t}\right)$. Furthermore, we predicted that both of these parameters influence prey survivorship independently and form a survivorship plane similar to Fig. 1. The results from our 3 laboratory experiments partially support this model.

Our results support the idea that $\mathrm{Sp} / \mathrm{Pr}$ influenced prey survivorship independently of $C_{t} / A_{t}$, and that the survivorship versus $\mathrm{Sp} / \mathrm{Pr}$ relationship has a decreasing sigmoid shape. In the first experiment the lowest $\mathrm{Sp} / \mathrm{Pr}$ value was 1.21 (Fig. 2a), which was not narrow enough to achieve an upper plateau of prey survivorship. It is obvious that with smaller spaces a plateau must exist, however, and we demonstrated this plateau in the third experiment with smaller Sp/Pr values (Fig. 4a).

Our results also support the idea that $C_{t} / A_{t}$ influenced prey survivorship independently of $\mathrm{Sp} / \mathrm{Pr}$; however, the survivorship versus $C_{t} / A_{t}$ relationship shape is not clear. In the first experiment the shape of the survivorship curve with $C_{t} / A_{t}$ in Fig. $2 b$ was either linear 
Table 3. Expt 2 results and structural parameters for the containers

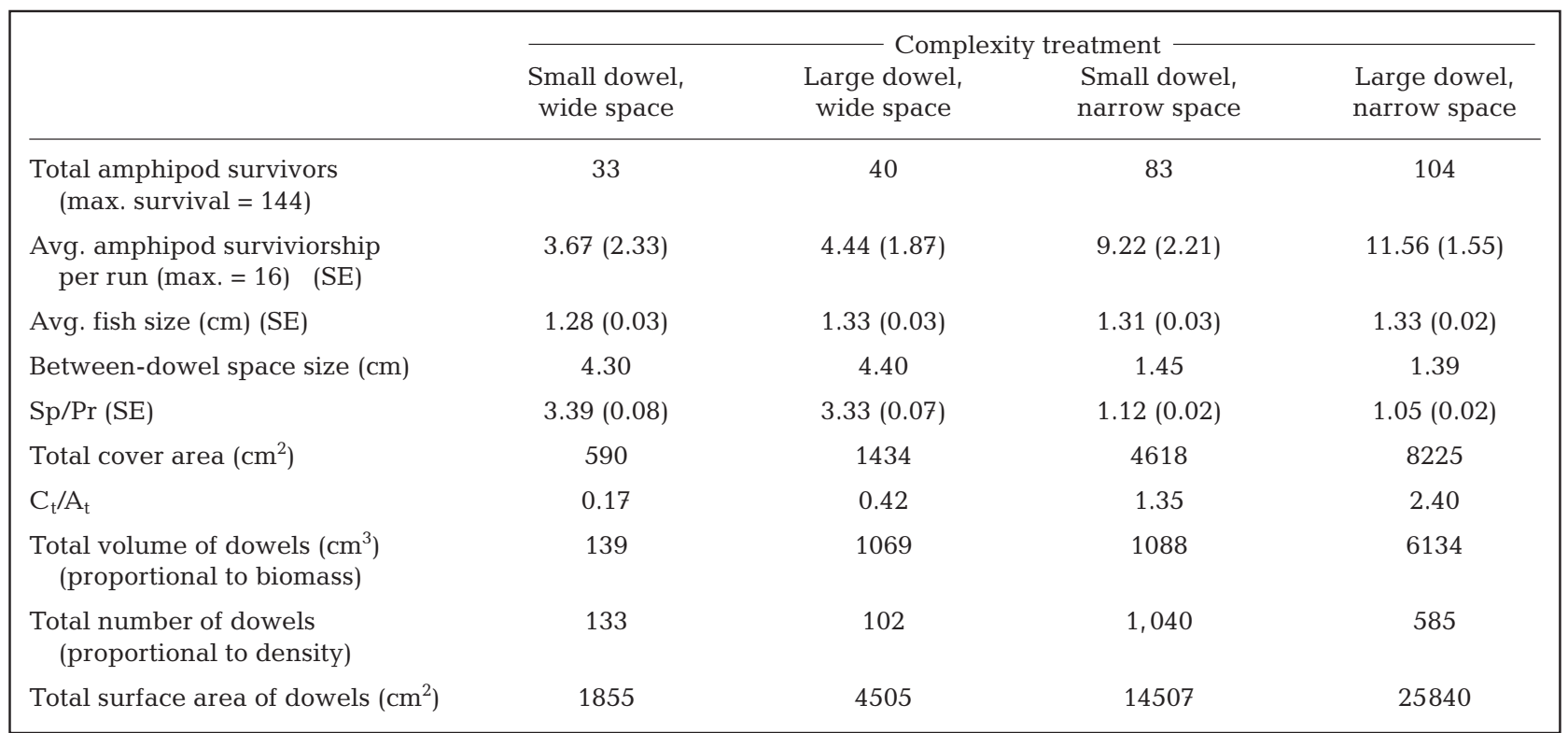

or slightly sigmoidal. The peak in the first derivative curve (Fig. 2d) that may imply a sigmoid relationship is fairly small, so the results, as shown (Fig. 2b), are probably simply linear. If the relationship is sigmoid, then the inflection point occurs at very low $C_{t} / A_{t}$ values (about 0.1). In the second experiment the relationship with $\mathrm{C}_{\mathrm{t}} / \mathrm{A}_{\mathrm{t}}$, as shown in Fig. $3 \mathrm{~b}$, is simply linear, with about 24 out of 96 amphipods surviving at $C_{t} / A_{t}=0$, if we extended a linear regression line through the $y$-axis.

There are 2 reasons that the relationship between $\mathrm{C}_{\mathrm{t}} / \mathrm{A}_{\mathrm{t}}$ and survivorship may be more hyperbolic than the results, as shown in Figs. $2 b \& 3 b$, indicate. In Fig. $2 b$, the higher $C_{t} / A_{t}$ treatments have high survivorship both because of high amounts of cover, and

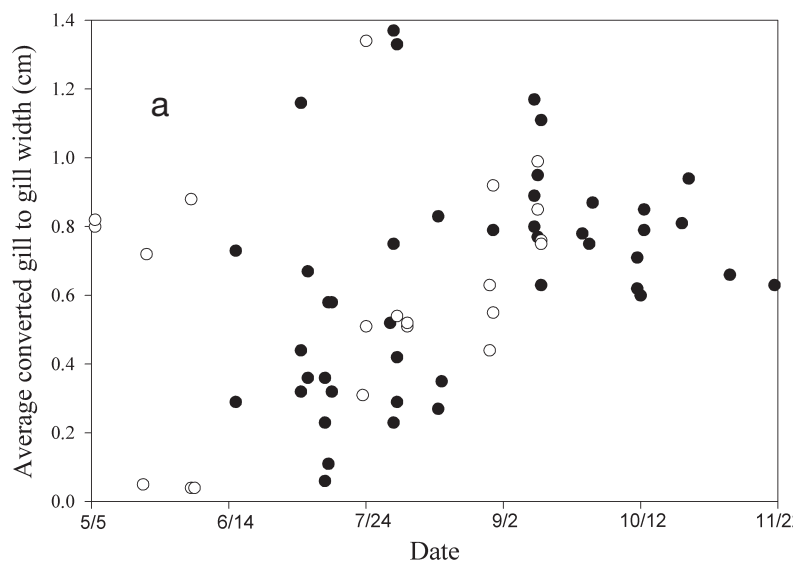

Table 4. Maximum likelihood log-linear model for Expt 3 Note: The interactions between fish size and prey survival and cover level and prey survival test the null hypotheses that these factors are independent. A significant result indicates non-independence

\begin{tabular}{|lccc|}
\hline Source & df & Chi-square & $p$ \\
\hline Fish size & 1 & 0.12 & 0.7268 \\
Cover area & 2 & 18.95 & 0.0001 \\
Survival & 1 & 19.55 & $<0.00005$ \\
Fish size $\times$ Survival & 1 & 5.94 & 0.0148 \\
Cover level $\times$ Survival & 2 & 140.29 & $<0.00005$ \\
Likelihood ratio & 4 & 6.10 & 0.1920 \\
\hline
\end{tabular}

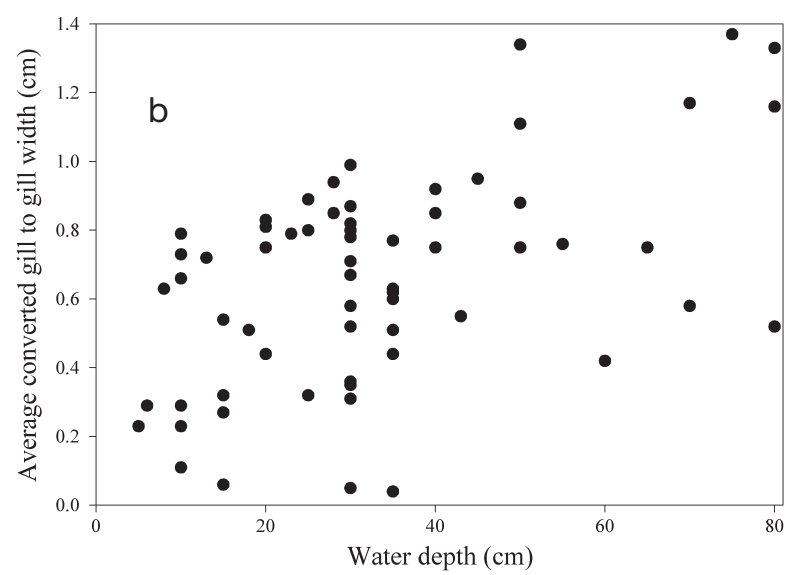

Fig. 5. (a) Data from drop ring samples, indicating a significant relationship between average Fundulus heteroclitus gill-to-gill width and date $(\mathrm{d} / \mathrm{mo})$. Filled circles are from 1995; open circles are from 1996. Gill-to-gill width (cm) was obtained by converting from the originally recorded wet weights (g). (b) The same drop ring samples, indicating a significant relationship between average $F$. heteroclitus gill-to-gill width and water depth at the time of sampling 
Table 5. Expt 3 results and structural parameters for the tanks

\begin{tabular}{|c|c|c|c|c|c|c|}
\hline & \multicolumn{6}{|c|}{ - Complexity treatment } \\
\hline & $\begin{array}{l}\text { Low cover, } \\
\text { small fish }\end{array}$ & $\begin{array}{l}\text { Medium cover, } \\
\text { small fish }\end{array}$ & $\begin{array}{l}\text { High cover, } \\
\text { small fish }\end{array}$ & $\begin{array}{l}\text { Low cover, } \\
\text { large fish }\end{array}$ & $\begin{array}{l}\text { Medium cover, } \\
\text { large fish }\end{array}$ & $\begin{array}{l}\text { High cover, } \\
\text { large fish }\end{array}$ \\
\hline $\begin{array}{l}\text { Total amphipod survivors } \\
\quad(\text { max. survival }=96)\end{array}$ & 19 & 43 & 88 & 22 & 65 & 92 \\
\hline $\begin{array}{l}\text { Avg. amphipod surviviorship } \\
\text { per run (max. = 16) }(\mathrm{SE})\end{array}$ & $3.17(1.45)$ & $7.17(1.47)$ & $14.67(0.76)$ & $3.67(2.32)$ & $10.83(1.72)$ & $15.33(0.33)$ \\
\hline Avg. fish size, cm (SE) & $1.24(0.02)$ & $1.25(0.02)$ & $1.25(0.02)$ & $1.58(0.03)$ & $1.55(0.02)$ & $1.55(0.03)$ \\
\hline Between-dowel space size (cm) & 4.30 & 2.33 & 1.45 & 4.30 & 2.33 & 1.45 \\
\hline $\mathrm{Sp} / \mathrm{Pr}(\mathrm{SE})$ & $3.48(0.05)$ & $1.92(0.04)$ & $1.16(0.02)$ & $2.73(0.04)$ & $1.50(0.02)$ & $0.94(0.02)$ \\
\hline Total cover area $\left(\mathrm{cm}^{2}\right)$ & 590 & 2020 & 4618 & 590 & 2020 & 4618 \\
\hline $\mathrm{C}_{\mathrm{t}} / \mathrm{A}_{\mathrm{t}}$ & 0.17 & 0.59 & 1.35 & 0.17 & 0.59 & 1.35 \\
\hline $\begin{array}{l}\text { Total volume of dowels }\left(\mathrm{cm}^{3} \text { ) }\right. \\
\text { (proportional to biomass) }\end{array}$ & 139 & 476 & 1088 & 139 & 476 & 1088 \\
\hline $\begin{array}{l}\text { Total number of dowels } \\
\text { (proportional to density) }\end{array}$ & 133 & 455 & 1040 & 133 & 455 & 1040 \\
\hline Total surface area of dowels $\left(\mathrm{cm}^{2}\right)$ & 1855 & 6347 & 14507 & 1855 & 6347 & 14507 \\
\hline
\end{tabular}

because of small space sizes, whereas in the lower $\mathrm{C}_{\mathrm{t}} / \mathrm{A}_{\mathrm{t}}$ treatments small space sizes certainly do not contribute to prey survivorship. Therefore, the higher $\mathrm{C}_{\mathrm{t}} / \mathrm{A}_{\mathrm{t}}$ treatments, particularly the highest 2 treatments, would have had lower survivorship if $C_{t} / A_{t}$ alone was contributing to prey survivorship, and the resultant relationship of survivorship versus $C_{t} / A_{t}$ in Fig. $2 b$ would be more hyperbolic. In Fig. $3 \mathrm{~b}$, if we assume low survivorship at low $C_{t} / A_{t}$, then the relationship between $C_{t} / A_{t}$ and survivorship conforms to a hyperbolic model. Low survivorship at low $C_{t} / A_{t}$ values may or may not be a valid assumption. We had no prey survivorship in all of our pilot studies with $C_{t} / A_{t}=0$ conducted before the first laboratory experiment, and during the first laboratory experiment only 6 amphipods survived out of 288 in the 3 lowest $C_{t} / A_{t}$ treatments. Clearly further work needs to be done to determine the shape of the relationship between $\mathrm{Ct}$ /At and prey survivorship.

Our results indicate that both $\mathrm{Sp} / \mathrm{Pr}$ and $\mathrm{C}_{\mathrm{t}} / \mathrm{A}_{\mathrm{t}}$ may influence prey survivorship, and that the observed patterns are not just because of a relationship between these 2 parameters. Both the Sp/Pr and $C_{t} / A_{t}$ terms are significant for the logistic regression, and the fit was remarkably good; both space size and dowel size were significant in the second experiment loglinear model, and both fish size and cover level were significant in the third experiment log-linear model. Overall, the survivorship results from the 3 experiments, when superimposed, followed a fairly consistent pattern, particularly with increasing $\mathrm{Sp} / \mathrm{Pr}$. Survivorship dropped rapidly after $\mathrm{Sp} / \mathrm{Pr}$ values of 1 (the predators could just barely maneuver), and reached a lower plateau. We suspect that the survivorship observed in Figs. 2a, 3a \& 4 a above $\mathrm{Sp} / \mathrm{Pr}$ values of approximately 2.2 was probably due to the cover alone, and not due to the fish's reduced maneuverability in smaller spaces, based on where the rapid increase, or threshold, begins in Figs. 2a \& 4a.

Our results appear to indicate that surface to volume ratios, volume of structures, biomass of structures, number of individual structures and density of individual structures were all poor predictors of prey survivorship when compared with $C_{t} / A_{t}$ and Sp/Pr. We make this conclusion because there is no clearly observable survivorship pattern with any of these other habitat

Table 6. ANCOVA for the field data (Cicchetti 1998), Spartina alterniflora form (short or tall) is the main factor, water depth and date are covariates, and fish gill-to-gill width is the dependent variable

\begin{tabular}{|lcccrc|}
\hline Source & df & Adj. SS & Adj. MS & $F$ & $p$ \\
\hline Date & 1 & 0.29174 & 0.29174 & 4.57 & 0.037 \\
Water depth & 1 & 0.88944 & 0.88944 & 13.93 & $<0.0005$ \\
S. alterniflora form & 1 & 0.10635 & 0.10635 & 1.67 & 0.202 \\
Error & 61 & 3.89506 & 0.06385 & & \\
\hline
\end{tabular}


parameters (Table 3). Measurements of these habitat parameters have been used to describe habitat complexity and refuge value in past predator/prey studies, but we suggest that there may be no direct mechanism by which predator foraging ability should be directly affected by these parameters. Certainly prey survivorship would tend to increase when these habitat parameters increase, but we suggest that $C_{t} / A_{t}$ and $S p / P r$ may better predict responses in prey survivorship to structure. These other parameters may be very useful in situations where $C_{t} / A_{t}$ and $S p / P r$ are difficult to measure, however.

We were not able to separate the effects of $C_{t} / A_{t}$ and total surface area. Habitats with high amounts of surface area would tend to have higher amounts of cover, and would also tend to have many small spaces that prey could hide in, and that predators could not maneuver through. However, structure with higher surface areas may provide worse refuge for prey, because of numerous small gaps that may allow a predator to see through the structure (Stoner 1982). We feel that total surface area may be a good predictor of prey survivorship in some cases, because higher total surface area is usually associated with higher $C_{t} / A_{t}$ values, and lower $\mathrm{Sp} / \mathrm{Pr}$ values.

The results of the field experiment demonstrate that the average fish size increased throughout the year from May to November (Fig. 5a), that fish size increased with increasing water depth on the marsh surface (Fig. 5b) and that different fish sizes did not utilize different Spartina alterniflora forms. The different $S$. alterniflora forms appeared to be different in complexity visually, and we confirmed that they were slightly different in average inter-structural space size. The distribution of fish sizes between the $2 S$. alterniflora forms did not respond to this difference in space size, however. Dividing the median inter-structural space size of both forms by the largest fish width caught by Cicchetti (1998) gives us an estimate of Sp/Pr for the largest fish. The $\mathrm{Sp} / \mathrm{Pr}$ for the tall form $S$. alterniflora is $4.2 / 1.4=3$, and for the short form is $3.3 / 1.4=2.4$. This is not a large difference in terms of $\mathrm{Sp} / \mathrm{Pr}$, and when compared to the results of the third experiment (Fig. 4a), these values are both on the lower plateau of $\mathrm{Sp} / \mathrm{Pr}$. Thus, we would not have expected to see any difference in Spartina form usage by large fish. The above $\mathrm{Sp} / \mathrm{Pr}$ values are for the largest fish caught, and smaller fish would be affected even less by any difference in space size. These field results are consistent with the lab results, and they serve to illustrate that measuring $\mathrm{Sp} / \mathrm{Pr}$ can be relatively simple in some habitat types. In other habitats $\mathrm{Sp} / \mathrm{Pr}$ and $\mathrm{C}_{\mathrm{t}} / \mathrm{A}_{\mathrm{t}}$ may be very difficult to measure.

These indices have other possible shortcomings as well. The effect that $\mathrm{Sp} / \mathrm{Pr}$ has on prey survival may depend upon how structures are physically arranged in space. Our tank experiments used uniformly spaced structures, for example, and our low complexity treatments provided uniformly poor refuges. Tanks having heterogeneous structural arrangements, with similar $\mathrm{Sp} / \mathrm{Pr}$ values, could have produced better overall survival if they provided a few small areas with excellent refuge values. Past research has shown that organisms can actively choose good refuge areas within heterogeneous habitats (Bell \& Westoby 1986). The effect that $\mathrm{Sp} / \mathrm{Pr}$ has on prey survivorship may also depend upon how rigid and strong the structures are within the habitat. The flexibility and strength of a structure may have important consequences for the survival of prey within the habitat (Coull \& Wells 1983). Flexible or weak structures may allow predators to push aside or break through the structure, thus gaining access to the prey. Flexible or weak structure may entangle or slow the progress of the predator, but it may not be able to halt the predator altogether. As a result, 2 habitats with identical $\mathrm{Sp} / \mathrm{Pr}$ and $\mathrm{C}_{\mathrm{t}} / \mathrm{A}_{\mathrm{t}}$ may produce different survivorship patterns based on the rigidity and strength of their structures. This would limit a researcher's ability to compare results between studies using these indices if the structures involved had very different flexibilities or strengths. Our complexity indices also do not account for cryptic coloration of prey organisms, or for animals adapted to look like some aspect of the habitat they live in. This can also increase prey survivorship dramatically, and there are innumerable examples of the adaptive significance of being a cryptic prey. These complexity indices also do not account for physical or chemical defenses of the structure that may indirectly benefit the prey organism (Duffy \& Hay 1991). Past indices of structural complexity as prey refuges have usually not accounted for the above factors either, although they can be of the utmost importance to prey survivorship.

In many cases we are interested in the refuge value of a habitat for a particular prey species, but the size of the potential predators may not be known. We propose a different index of complexity for these circumstances: the average size of the inter-structural spaces relative to the size of the prey of interest $(\mathrm{Sp} / \mathrm{Py})$. The size of the prey in this case is the largest size that would limit the prey's ability to move through the habitat spaces. If the prey, on average, can move through the spaces within the habitat but any predators larger than the prey cannot, survivorship should be uniformly high. Survivorship would then decrease rapidly with increasing $\mathrm{Sp} / \mathrm{Py}$, because the number of predators that can fit through the habitat spaces accumulates, and the maneuverability of the predators that can fit through the spaces would also increase. At very high $\mathrm{Sp} / \mathrm{Py}$ ratios only the very largest of the potential 
predators would be impeded, and survivorship would be uniformly low. This would form a sigmoid relationship similar to $\mathrm{Sp} / \mathrm{Pr}$.

In some habitats, the space sizes may be too small for the prey to move through effectively $(\mathrm{Sp} / \mathrm{Py}<1)$, and prey survivorship may be low because the spaces within the habitat cannot be used as refuges. For example, Moksnes et al. (1998) found that juvenile shore crabs Carcinus maenas generally survived well in ephemeral macroalgae, but that survivorship differed between macroalgal species. Survivorship was high in algae of medium complexity, but low in foliose and finely filamentous algae. This may have occurred because the foliose and finely filamentous algal space sizes, relative to the size of the crabs, were too large to exclude predators, and too small to be used as refuges, respectively (P.-O. Moksnes pers. comm.). Hacker \& Steneck (1990) demonstrated that amphipods Gammarellus angulosus are sensitive to the space sizes within a habitat relative to their body size (Sp/Py). They also demonstrated that organisms may prefer habitats that are not 'too complex', i.e. habitats with spaces that are too small to move through. Other researchers have found that the space sizes within habitats, relative to both predator and prey sizes, can have important implications for the species involved (Steger 1987, Hixon \& Beets 1989, Friedlander \& Parrish 1998).

We feel that our complexity indices may be useful for future ecological work, because they may allow quantitative comparison of results between studies. Our results and model support the past findings of other researchers working with various predator/prey/habitat systems, although these researchers measured complexity in numerous different ways. Main (1987) and Savino \& Stein (1982) both demonstrated the importance of cover to a prey species' survival. They also illustrated that prey species may have adaptive behaviors that allow them to maximize the utility of the cover present within a habitat. Ryer (1988) found that the attack probabilities of large pipefish Syngnathus fuscus were affected by increased habitat complexity, whereas the rates of small $S$. fuscus were not affected. Ryer (1988) attributed this difference to the inability of large fish to fit their mouths into the small gaps produced by the wider, more complex, artificial seagrass leaves. Our research supports the results of these studies, as we also demonstrate the importance of total cover and fish size relative to inter-structural space size.

Schulman (1996), Lipcius et al. (1998) and Graham et al. (1998) have found hyperbolic relationships of prey survivorship with increasing amounts of cover. Lipcius et al. (1998) found steeply hyperbolic survivorship curves for small and large Caribbean spiny lobsters Panulirus argus with increasing algal biomass $\mathrm{m}^{-2}$.
Similarly, Schulman (1996) found hyperbolic survivorship curves for large juvenile blue crabs Callinectes sapidus with increasing artificial seagrass densities. Graham et al. (1998), in an experiment remarkably similar to ours, found that amphipod survivorship increased in a hyperbolic manner with increasing artificial eelgrass densities when exposed to Fundulus heteroclitus. Again, our model predicts hyperbolically increasing survivorship with increasing $C_{t} / A_{t}$ although it is unclear whether our results support their findings or our model.

Gotceitas \& Colgan (1989) and Coull \& Wells (1983) (see also sources therein) found threshold relationships of complexity with predator foraging success and prey survival, respectively. In contrast, Nelson \& Bonsdorff (1990) found a linear relationship between complexity and number of prey eaten. The results of Nelson \& Bonsdorff (1990) may have been masking an actual threshold relationship of complexity and number of prey eaten in their experiment, however (E. Bonsdorff pers. comm.).

In Nelson \& Bonsdorff's (1990) Fig. 3, one cannot distinguish between 2 different causes of prey survivorship in the lowest and medium complexity treatments, because they used more prey (15 fish) than the predators could eat (approximately 6.5 fish) within the experimental time period. Prey may or may not be surviving in the lowest complexity treatments due to predator satiation (predators will not eat more prey), and prey may or may not be surviving in the medium complexity treatments due to the physical structure (predators cannot catch more prey). If the predators could have physiologically eaten all of the prey in the lowest complexity treatments, the mean number of prey eaten per tank for these treatments may have been higher, relative to the medium complexity treatments, and may have formed an upper plateau at these complexity levels. Mean number of prey eaten per tank may then have dropped rapidly to the intermediate numbers of prey eaten per tank observed in the medium complexity treatments, where survivorship may have been due to the structure. Finally their results indicate a lower plateau of mean number of prey eaten per tank with their most complex treatments (Nelson \& Bonsdorff 1990; their Fig. 3). Overall, these hypothetical results would have produced a threshold relationship of complexity and mean number of prey eaten per tank. However, they could not have achieved an upper plateau of mean number of prey eaten per tank as they used too many prey fish, and thus could not have detected a threshold relationship if it had existed.

Our results, and our model of survivorship versus $\mathrm{Sp} / \mathrm{Pr}$ support the threshold hypothesis, and the results of Gotceitas \& Colgan (1989), Coull \& Wells (1983) and 
other studies referred to by Coull \& Wells (1983). We feel that structural habitat complexity thresholds should exist in nature with increasing $\mathrm{Sp} / \mathrm{Pr}$, and our laboratory results support this idea.

\section{CONCLUSIONS}

We devised 2 new indices of structural habitat complexity for assessing the effect of structural complexity on predator foraging success. These indices are: (1) the total amount of cover within a given habitat divided by the habitat area $\left(C_{t} / A_{t}\right)$, and (2) the average size of the inter-structural spaces within the habitat divided by the size of the predator $(\mathrm{Sp} / \mathrm{Pr}) . \mathrm{C}_{\mathrm{t}} / \mathrm{A}_{\mathrm{t}}$ measures the amount of cover within a habitat that prey species can hide behind, and Sp/Pr measures the extent to which predator maneuverability is reduced within a habitat.

From first principles we predicted that prey survivorship should increase hyperbolically with increasing $\mathrm{C}_{t} / \mathrm{A}_{\mathrm{t}}$, and should decrease sigmoidally with increasing $\mathrm{Sp} / \mathrm{Pr}$. We also predicted that both of these can independently influence prey survivorship, forming a survivorship plane. We tested these hypotheses in 3 laboratory experiments and 1 field experiment, and our results support our model for $\mathrm{Sp} / \mathrm{Pr}$ and are suggestive for $C_{t} / A_{t}$.

$\mathrm{Sp} / \mathrm{Pr}$ and $\mathrm{C}_{t} / \mathrm{A}_{\mathrm{t}}$ may prove to be useful in future studies involving predator/prey/habitat relationships. These indices of complexity are dimensionless, they can be applied across all habitat types, and they may allow easier comparison of results between habitat types. In addition, we feel that these indices of complexity directly measure how structural complexity interferes with a predator's foraging ability.

Acknowledgements. We would like to thank Dr Romuald Lipcius, Dr Emmett Duffy, Dr Erik Bonsdorff, Mary Ann Vogelbein, Dr Kevin Hovel and Dr Robert Orth for their help on this project, and their helpful comments on the manuscript. Thanks also to T. V. Padma. We would also like to thank the anonymous reviewers for their helpful comments. A.B. was funded by a VIMS fellowship, and a STAR fellowship from the EPA. This project was funded by the Lerner-Gray Fund for Marine Research, from the American Museum of Natural History. Thank you. This is VIMS contribution no. 2336.

\section{LITERATURE CITED}

Agresti A (1990) Categorical data analysis. John Wiley and Sons, New York

Aronson RB (1986) Life history and den ecology of Octopus briareus Robson in a marine lake. J Exp Mar Biol Ecol 95: $37-54$

Bell J, Westoby M (1986) Abundance of macrofauna in dense seagrass is due to habitat preference, not predation. Oecologia 68:205-209

Bell SS (1985) Habitat complexity of polychaete tube caps: influence of architecture on dynamics of a meioepibenthic assemblage. J Mar Res 43:647-657

Bousfield EL (1973) Shallow water gammaridean amphipoda of New England. Cornell University Press, Ithaca, NY

Cicchetti G (1998) Habitat use, secondary production, and trophic export by salt marsh nekton in shallow waters. VIMS dissertation. College of William and Mary, Gloucester Point, VA

Coull BC, Wells JBJ (1983) Refuges from fish predation: experiments with phytal meiofauna from the New Zealand rocky intertidal. Ecology 64:1599-1609

Dean RL, Connell JH (1987) Marine invertebrates in an algal succession. III. Mechanisms linking habitat complexity with diversity. J Exp Mar Biol Ecol 109:249-273

Diehl S (1988) Foraging efficiency of three freshwater fishes: effects of structural complexity and light. Oikos 53: 207-214

Dickman CR (1992) Predation and habitat shift in the house mouse, Mus domesticus. Ecology 73:313-322

Duffy JE, Hay ME (1991) Food and shelter as determinants of food choice by an herbivorous marine amphipod. Ecology 72:1286-1298

Friedlander AM, Parrish JD (1998) Habitat characteristics affecting fish assemblages on a Hawaiian coral reef. J Exp Mar Biol Ecol 224:1-30

Gorham JC, Alevizon WS (1989) Habitat complexity and the abundance of juvenile fishes residing on small scale artificial reefs. Bull Mar Sci 44:662-665

Gotceitas V, Colgan P (1989) Predator foraging success and habitat complexity: quantitative test of the threshold hypothesis. Oecologia 80:158-166

Graham S, Davis J, Deegan L, Cebrian J, Hughes J, Hauxwell $J$ (1998) Effect of eelgrass (Zostera marina) density on the feeding efficiency of mummichog (Fundulus heteroclitus). Biol Bull 195:241-243

Hacker SD, Steneck RS (1990) Habitat architecture and the abundance and body-size-dependent habitat selection of a phytal amphipod. Ecology 71:2269-2285

Heck KL Jr, Crowder LB (1991) Habitat structure and predator-prey interactions in vegetated aquatic systems. In: Bell SS, McCoy ED, Mushinsky HR (eds) Habitat structure, the physical arrangement of objects in space. Chapman \& Hall, New York, p 281-299

Heck KL Jr, Wetstone GS (1977) Habitat complexity and invertebrate species richness and abundance in tropical seagrass meadows. J Biogeogr 4:135-142

Hicks GRF (1985) Meiofauna associated with rocky shore algae In: Moore PG, Seed R (eds) The ecology of rocky coasts. Hoder \& Stoughton, London, p 36-64

Hixon MA, Beets JP (1989) Shelter characteristics and Caribbean fish assemblages: experiments with artificial reefs. Bull Mar Sci 44:666-680

James PL, Heck KL Jr (1994) The effects of habitat complexity and light intensity on ambush predation within a simulated seagrass habitat. J Exp Mar Biol Ecol 176:187-200

Jordan F, DeLeon CJ, McCreary AC (1996) Predation, habitat complexity, and distribution of the crayfish Procambarus alleni within a wetland mosaic. Wetlands 16:452-457

Lipcius RN, Eggleston DB, Miller DL, Luhrs TC (1998) The habitat-survival function for Carribean spiny lobster: an inverted size effect and non-linearity in algal and seagrass habitats. Mar Freshw Res 149:807-816

Main K (1987) Predator avoidance in seagrass meadows: prey behavior, microhabitat selection and cryptic coloration. Ecology 68:170-180

McCoy ED, Bell SS (1991) Habitat structure: the evolution and diversification of a complex topic. In: Bell SS, McCoy ED, 
Mushinsky HR (eds) Habitat structure, the physical arrangement of objects in space. Chapman \& Hall, New York, p 3-27

Moksnes PO, Pihl L, Van Montfrans J (1998) Predation on postlarvae and juveniles of the shore crab Carcinus maenas: importance of shelter, size and cannibalism. Mar Ecol Prog Ser 166:211-225

Nelson WG, Bonsdorff E (1990) Fish predation and habitat complexity: are complexity thresholds real? J Exp Mar Biol Ecol 141:183-194

O'Connor NA (1991) The effects of habitat complexity on the macroinvertebrates colonising wood substrates in a lowland stream. Oecologia 85:504-512

Orth RJ, Heck KL Jr, Van Montfrans J (1984) Faunal communities in seagrass beds: a review of the influence of plant structure and prey characteristics on predator-prey relationships. Estuaries 7:339-350

Pierce CL (1988) Predator avoidance, microhabitat shift, and risk-sensitive foraging in larval dragonflies. Oecologia 77: 81-90

Ruiz GM, Hines AH, Posey MH (1993) Shallow water as a refuge habitat for fish and crustaceans in non-vegetated estuaries: an example from Chesapeake Bay. Mar Ecol Prog Ser 99:1-16

Ryer C (1988) Pipe fish foraging: effects of fish size, prey size and altered habitat complexity. Mar Ecol Prog Ser 48: $37-45$

Editorial responsibility: Kenneth Heck (Contributing Editor), Dauphin Island, Alabama, USA
Savino J, Stein RA (1982) Predator-prey interaction between large mouth bass and bluegills as influenced by simulated, submerged vegetation. Trans Am Fish Soc 111:255-266

Schneider KJ (1984) Dominance, predation and optimal foraging in white-throated sparrow flocks. Ecology 65: 1820-1827

Schulman J (1996) Habitat complexity as a determinant of juvenile blue crab survival. VIMS dissertation. College of William and Mary, Gloucester Point, VA

Steger R (1987) Effects of refuges and recruitment on gonodactylid stomatopods, a guild of mobile prey. Ecology 68: 1520-1533

Stokes M, Davis C, Koch G (1995) Categorical data analysis using the SAS system. SAS Institute Inc, Cary, NC

Stoner A (1980) Perception and choice of substratum by epifaunal amphipods associated with seagrasses. Mar Ecol Prog Ser 3:105-111

Stoner A (1982) The influence of benthic macrophytes on the foraging behavior of pinfish, Lagodon rhomboides (Linneus). J Exp Mar Biol Ecol 58:271-284

van Dolah RF (1978) Factors regulating the distribution and population dynamics of the amphipod Gammarus palustris in an intertidal salt marsh community. Ecol Monogr 48: 191-217

Werner EE, Hall DJ (1988) Ontogenetic habitat shifts in bluegill: the foraging rate-predation risk trade off. Ecology 69:1352-1366

Submitted: March 25, 1999; Accepted: March 2, 2000 Proofs received from author(s): October 9, 2000 